\title{
Seronegative Maternal Ocular Myasthenia Gravis and Delayed Transient Neonatal Myasthenia Gravis
}

\author{
Courtney Townsel, MD ${ }^{1}$ Rebecca Keller, MD ${ }^{1}$ Kendall Johnson, MD ${ }^{2}$ Naveed Hussain, MD² \\ Winston A. Campbell, MD ${ }^{1}$
}

${ }^{1}$ Division of Maternal Fetal Medicine, University of Connecticut School of Medicine, Farmington, Connecticut

2 Division of Neonatology, Connecticut Children's Medical Center, Farmington, Connecticut
Address for correspondence Courtney Townsel, MD, Division of Maternal Fetal Medicine, UCONN Health, 263 Farmington Avenue, Farmington, CT 06030-2946 (e-mail: townsel@uchc.edu).

Am J Perinatol Rep 2016;6:e133-e136.

\begin{abstract}
Keywords

- ocular myasthenia gravis

- pregnancy

- transient neonatal myasthenia gravis

Background Myasthenia gravis (MG) is an autoimmune disorder with fluctuating muscle weakness, divided into generalized and localized (ocular) forms. Maternal antibodies to acetylcholine receptors cross the placenta and may cause transient neonatal myasthenia gravis (TNMG). We present a case of seronegative maternal ocular MG and delayed TNMG.

Case A 29-year-old G3P1011 underwent cesarean birth of a male infant who developed oxygen desaturation requiring supplemental oxygen on day of life (DOL) 3. Based on the clinical course and after exclusion of other diagnoses, the infant was diagnosed with TNMG. Infant's condition improved spontaneously and he was weaned off supplemental oxygen and discharged home on DOL 12.

Conclusion Infants born to mothers with seronegative localized (ocular) MG are also susceptible to TNMG which may be late in onset.
\end{abstract}

Myasthenia gravis (MG) is an autoimmune disorder mediated by immunoglobulin $G$ autoantibodies to components of the postsynaptic neuromuscular junction (NMJ) characterized by fluctuating fatigable skeletal muscle weakness. ${ }^{1}$ There are two forms of MG: generalized and localized (ocular). MG affects women in their second or third decades of life overlapping with child-bearing age. ${ }^{2}$ During pregnancy, a third of pregnant women with MG have exacerbations, and the remaining twothirds have no change or remission of symptoms. ${ }^{3}$ Antiacetylcholine receptor (anti-AChR) antibodies are found in 80 to $90 \%$ of patients with generalized MG and 50 to $70 \%$ of patients with localized (ocular) MG. ${ }^{3}$ The antibody concentration correlates poorly with clinical status. ${ }^{4}$ Maternal antibodies can freely cross the placenta and cause short-term myasthenialike symptoms such as respiratory distress, poor feeding, and flaccid tone in the neonate. When these symptoms are present following delivery, this is referred to as transient neonatal myasthenia gravis (TNMG). This occurs in 10 to $20 \%$ of neonates born to women with generalized MG. ${ }^{3,5}$ For affected neonates, this can be a life-threatening condition, causing death by aspiration or respiratory failure. ${ }^{6}$ TNMG symptoms usually appear within 48 hours of birth and may persist for up to 3 months. ${ }^{6}$ The largest case series of clinically confirmed MG in pregnancy by Djelmis et al reported on 69 cases, categorizing patients into localized (ocular) or generalized (mild, moderate, severe) disease. This report documented the adverse maternal and neonatal outcomes in cases of generalized but not localized MG. ${ }^{7}$ We conducted a PubMed literature search using search terms "ocular myasthenia gravis," "pregnancy," and "neonatal." This did not identify any cases of TNMG in mothers with ocular MG. To our knowledge, this appears to be the first case of TNMG occurring in a neonate born to a mother specifically with seronegative ocular MG whose disease was in remission prior to and throughout pregnancy. received

December 14, 2015 accepted after revision January 27, 2016
DOI http://dx.doi.org/ 10.1055/s-0036-1579624. ISSN 2157-6998.
Copyright $\odot 2016$ by Thieme Medical Publishers, Inc., 333 Seventh Avenue, New York, NY 10001, USA. Tel: +1(212) 584-4662.
License terms

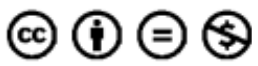




\section{Case}

A 29-year-old G3P1011 Caucasian patient was referred to our perinatal practice due to a history of pseudotumor cerebri, on acetazolamide, and ocular MG with thymic hyperplasia. Her seronegative ocular MG was diagnosed a year prior to this pregnancy after she presented with fatigable left eyelid ptosis. On evaluation, she had a positive ice test, absence of antiAChR antibodies, and absence of anti-striated muscle antibodies in her serum, which classified her as double seronegative MG (dSN-MG). Thymic hyperplasia was diagnosed on mediastinal positron emission tomography-computed tomography. A scheduled thymectomy was cancelled due to the diagnosis of a 6 -week intrauterine gestation. She underwent a dilatation and curettage at 8 weeks 6 days for a missed abortion. She conceived 2 months later and initiated her prenatal care with our practice at 12 weeks of gestation.

During this pregnancy, her neuro-ophthalmologist maintained her on acetazolamide $500 \mathrm{mg}$ daily for pseudotumor cerebri, and butalbital, acetaminophen, and caffeine in combination as needed for headache. Her last ocular MG flare was at the time of her initial diagnosis. The pregestational remission of her ocular MG continued throughout the pregnancy. Prenatal laboratory testing, genetic screening, and second-trimester anatomy ultrasound were within normal limits. An interval growth ultrasound at 28 weeks revealed normal amniotic fluid and fetal tone, and appropriate fetal growth. At her prenatal visits, the patient always reported normal fetal movement.

At 39 weeks of gestation, due to worsening papilledema from her pseudotumor cerebri, her neuro-ophthalmologist recommended the patient be delivered by cesarean birth. She was scheduled for a primary cesarean delivery. Upon hospital admission, her vital signs were within normal limits and the fetal heart rate tracing was reactive. The patient underwent a primary cesarean delivery under spinal anesthesia. She was delivered of a vigorous male infant whose birth weight was $7 \mathrm{lb} 5 \mathrm{oz}$ (3,325 g), and Apgar scores were 8 and 9 at 1 and 5 minutes of life, respectively. The delivery was complicated by a uterine inversion that was resolved with manual replacement. The intraoperative blood loss was estimated as 1,200 $\mathrm{mL}$. On postoperative day 3 , she reported lightheadedness. Her hematocrit decreased from 31.9 preoperatively to 20.7 postoperatively. She declined recommended blood transfusion, requesting only oral iron therapy. Her symptoms improved with intravenous fluid administration, and she was discharged in stable condition on postoperative day 4 on oral iron supplementation.

Due to the uncertainty of the neonatal risk in cases of localized MG, the on-service neonatologist recommended admission to the neonatal intensive care unit (NICU) for 48 hours of monitoring. The infant's initial exam demonstrated a vigorous infant with normal tone, no respiratory distress, and a strong suck. A capillary blood gas at 1 hour of life was $\mathrm{pH}$ $7.21 / \mathrm{CO}_{2} 53 / \mathrm{O}_{2} 42 /$ base excess (BE) -8 . This metabolic acidosis was thought to be consistent with maternal use of acetazolamide during pregnancy. ${ }^{8}$ The acidosis improved with the neonate just on room air. On the third day of life
(DOL), he had intermittent, self-recovered desaturations to 88 to $89 \%$ as well as periodic shallow breathing. On DOL 5 , his oxygen saturation was persistently low with persistent readings of $86 \%$, requiring $2 \mathrm{~L} / \mathrm{min}$ nasal cannula with $\mathrm{FiO}_{2}$ of $35 \%$ to maintain saturations $>90 \%$. A chest X-ray (CXR) showed the lungs were well expanded with mildly prominent vascular markings. An arterial blood gas was $\mathrm{pH} 7.37 / \mathrm{CO}_{2} 55 / \mathrm{O}_{2} 33 / \mathrm{BE}$ +5 . This was consistent with a chronic respiratory acidosis with renal compensation. A pediatric cardiology consult was obtained. The ultrasound echocardiogram of the heart did not find any congenital cardiac disease, but raised a question of an atrial septal defect versus persistent foramen ovale. $\mathrm{A} \mathrm{pH}$ probe and pneumogram demonstrated no significant reflux, central apnea, or periodic breathing. However, it did show intermittent hypoventilation. A repeat CXR at the time of the pneumogram showed increasingly hazy lung fields, consistent with microatelectasis. An ultrasound of the diaphragm showed normal excursion with no weakness or paralysis. A neurology consult was obtained and a complete neurologic exam demonstrated normal strength and tone with no deficits. The pediatric neurologist's assessment was that the infant's respiratory symptoms were most consistent with TNMG. Recommendations were made to send AChR antibodies and anti-muscle-specific tyrosine kinase (anti-MuSK) antibodies, both of which were negative. On DOL 12, his oxygen requirement decreased to an $\mathrm{FiO}_{2}$ of $21 \%$. The nasal cannula was discontinued on DOL 13. Repeat blood gas on room air was $\mathrm{pH} 7.46 / \mathrm{CO}_{2} 44 / \mathrm{O}_{2} 38 / \mathrm{BE}+7$, consistent with an improving respiratory acidosis. A repeat CXR also showed clear lungs fields. He was monitored for 4 days off respiratory support during which time he had no desaturations and was discharged home on DOL 17.

Maternal anti-AChR and anti-MuSK were repeated 3 months postpartum and were again negative.

\section{Discussion}

The neonatal diagnosis of TNMG was one of exclusion and based on the clinical presentation. Given that this is a case of maternal seronegative MG, it is not expected that the infant would have positive antibodies. About $15 \%$ of MG patients present with only localized or ocular disease and half of these patients have no detectable antibodies. ${ }^{9}$ Furthermore, there were several factors that demonstrated the infant was experiencing hypoventilation. It was presumed this was due to TNMG as there was no other etiology found despite extensive work-up. Other supporting factors were the mother's diagnosis of MG and the transient neonatal hypoventilation. On exam, the infant was noted to have periods of shallow breathing, blood gas was consistent with chronic $\mathrm{CO}_{2}$ retention with renal compensation, the CXR showed microatelectasis, and the pneumogram showed periods of hypoventilation. The pediatric neurology consult agreed that the presentation was most consistent with TNMG.

TNMG can present with respiratory difficulty which may be mild presenting with hypoventilation to severe requiring mechanical ventilation. Signs of TNMG usually occur within the first 48 hours of life. ${ }^{6}$ There is a paucity of information in 
the literature about localized (ocular) MG and neonate risk for TNMG. The incidence of MG in pregnancy is 1:20,000, and it remains an important disorder for obstetricians and neonatologists to be familiar with as it may pose challenges for maternal and neonatal care. ${ }^{3}$ To our knowledge, this is the first report of a mother in remission with double seronegative localized (ocular) MG who delivered an infant that developed TNMG more than 48 hours after birth. Recommendations for medical care for myasthenic patients are that it should be the same as in nonpregnant patients except for avoidance of magnesium sulfate, which can precipitate a myasthenic crisis, and use of muscle relaxant anesthetic drugs. ${ }^{6}$ A search of ACOG publications for guidance in managing MG in pregnancy yielded recommendations in Practice Bulletin \#127 Management of Preterm Labor, and patient safety checklists for Preeclampsia and Eclampsia to avoid the use of magnesium sulfate in management of preterm labor or hypertensive disorders in myasthenic patients (www.acog.org). The United Kingdom multispecialty working group recently published a best practice guideline for management of MG in pregnancy. ${ }^{10}$ The guideline provides expanded specific information on medication safety, maternal management, pregnancy monitoring, and neonatal care. The publications reviewed for the guidelines did not differentiate outcomes for generalized versus localized (ocular) MG. Only one study of the eight case series included had clinically confirmed cases of maternal MG, the others relied on discharge coding to identify mothers with MG. The guidelines recommend a neonatal observation period of at least 2 days. However, our neonate did not develop symptoms until 3 days following delivery.

Localized (ocular) MG is considered a milder form of the disease as compared with generalized MG, which ranges from mild disease causing ptosis and fatigue to severe disease requiring intubation. However, the pathophysiology of both localized and generalized disease is the same. ${ }^{11}$ It is important to note that of patients presenting with localized MG,
33 to $66 \%$ may go on to develop generalized MG. ${ }^{12}$ Based on the localized nature of our patient's disease, her antepartum care plan did not include a recommendation for neonatal surveillance in the NICU. As in our case, carrying the diagnosis of localized MG may reduce clinician anticipation for poor pregnancy or neonatal outcomes. ${ }^{11}$

MG occurs as a result of a decreased number of AChR and flattening of postsynaptic folds, which decrease the efficiency of neuromuscular transmission. This subsequently results in weakened muscle contractions. The changes are felt to be caused by anti-AChR antibodies. ${ }^{6}$ Over the past decade, evolving research in the area of postsynaptic receptor antibodies, beyond anti-AChR, has revealed new NMJ targets that may hold the key to a definitive diagnosis in $\mathrm{dSN}-\mathrm{MG}$ patients. Low-density lipoprotein receptor-related protein 4 (LRP4), MuSK, and collagen-Q (ColQ) have been shown to form a conglomerate at the NMJ that interacts with agrin, which is secreted by nerves and binds the LRP4:MuSK:ColQ subunit to promote intracellular signaling ( - Fig. 1 ). ${ }^{13}$ The MuSK-mediated pathway is responsible for aggregation of AChRs in the junctional plasma membrane, while ColQ is responsible for anchoring acetylcholinesterase which is central to removal of acetylcholine at the NMJ and normal muscular functioning. ${ }^{13}$ Thus, there are several NMJ targets that when blocked or activated may contribute to symptoms of MG. Zisimopoulou et al recently found that the frequency of anti-LRP4 in dSNMG patients ranges from 7 to $35 \%$, and more specifically $27 \%$ (15/55 patients) of dSN ocular MG patients had anti-LRP4. ${ }^{14} \mathrm{~A}$ smaller case series by Tsivgoulis et al supports the phenotype of dSN-MG with LRP4 antibodies presenting with isolated localized disease. ${ }^{15}$ Agrin and ColQ autoantibodies are among the next targets under further investigation as determinants in seronegative MG. Further investigation of these antibodies may lead to definitive diagnosis of MG in patients and TNMG in their neonates. While we were able to test for MuSK antibodies, laboratory testing for these newer targets are not yet readily available for routine patient evaluation.
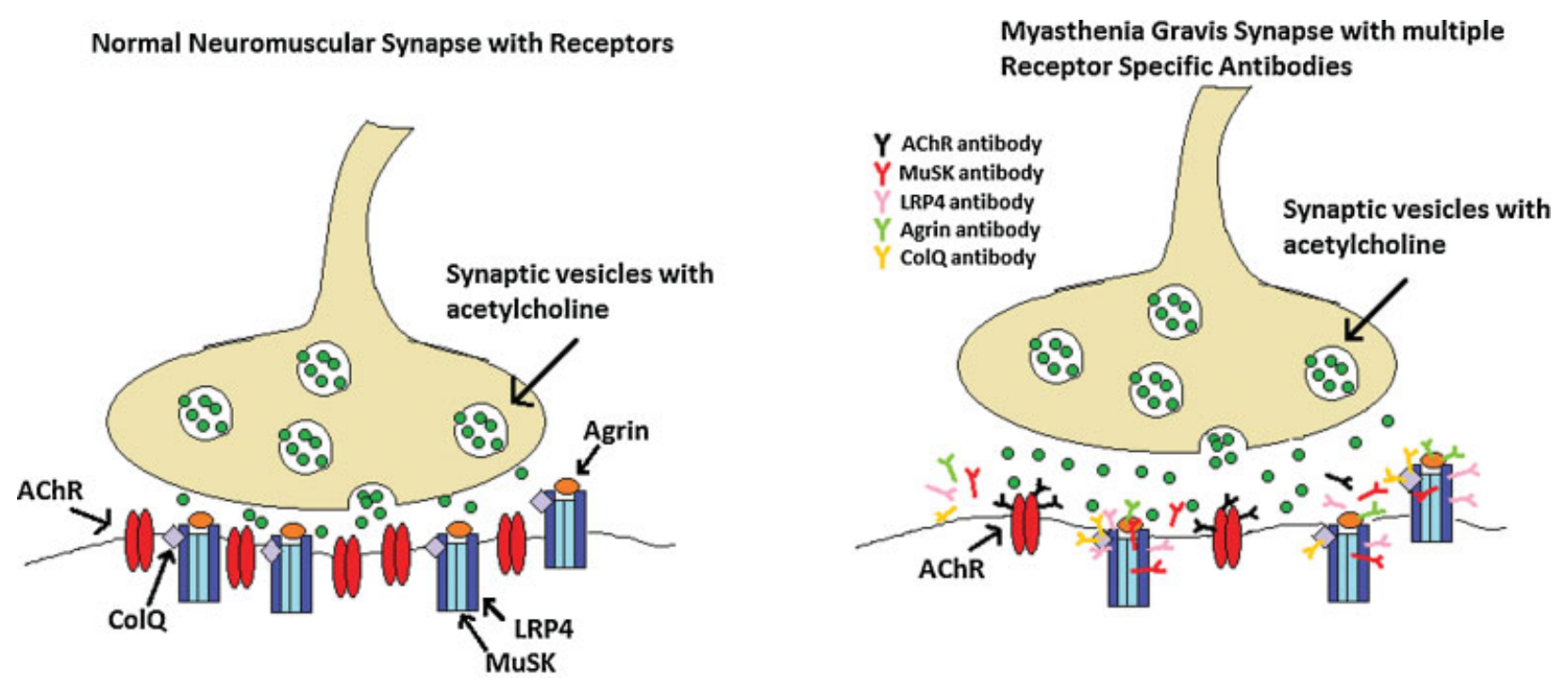

Fig. 1 Postsynaptic neuromuscular junction receptors and receptor antibodies causing myasthenia gravis. AChR, acetylcholine receptor; ColQ, collagen Q; LRP4, lipoprotein receptor-related protein 4; MuSK, muscle-specific tyrosine kinase receptor. 
Additionally, the neonatal course in our case was further complicated by maternal use of acetazolamide during pregnancy, with subsequent neonatal metabolic acidosis. The relationship between maternal acetazolamide use and subsequent neonatal metabolic acidosis is poorly characterized in the literature, with few cases reported on this occurrence. 8

This case has important clinical management value to obstetricians and pediatricians who care for pregnant myasthenic patients and their newborn infants. Even mothers with the localized (ocular) subtype of MG remain at risk of delivering an infant who may develop TNMG. The absence of AChR antibody in maternal serum does not imply the infant is not at risk for TNMG. There could be maternal antibodies to other postsynaptic neuromuscular receptors that can cause MG. Prolonged neonatal monitoring beyond 48 hours is warranted as some neonates may not develop symptoms until 72 hours following delivery.

\section{References}

1 Drachman DB. Myasthenia gravis. N Engl J Med 1994;330(25): 1797-1810

2 Plauché WC. Myasthenia gravis in mothers and their newborns. Clin Obstet Gynecol 1991;34(1):82-99

3 Kalidindi M, Ganpot S, Tahmesebi F, Govind A, Okolo S, Yoong W. Myasthenia gravis and pregnancy. J Obstet Gynaecol 2007;27(1): 30-32

4 Da Silva FC, do Cima LC, de Sá RAM. Myasthenia gravis and pregnancy. In: Minagar A ed. Neurological Disorders and Pregnancy. Burlington, MA: Elsevier; 2011:55-68
5 Papazian O. Transient neonatal myasthenia gravis. J Child Neurol 1992;7(2):135-141

6 Creasy RK, Resnik R, Greene MF, Iams JD, Lockwood CJ; Maternal Fetal Medicine: Principles and Practice. Ch 52, pg 1108-1109. Philadelphia, PA. Seventh edition. 2014

7 Djelmis J, Sostarko M, Mayer D, Ivanisevic M. Myasthenia gravis in pregnancy: report on 69 cases. Eur J Obstet Gynecol Reprod Biol 2002;104(1):21-25

8 Merlob P, Litwin A, Mor N. Possible association between acetazolamide administration during pregnancy and metabolic disorders in the newborn. Eur J Obstet Gynecol Reprod Biol 1990;35(1): 85-88

9 Berrih-Aknin S, Frenkian-Cuvelier M, Eymard B. Diagnostic and clinical classification of autoimmune myasthenia gravis. J Autoimmun 2014;48-49:143-148

10 Norwood F, Dhanjal M, Hill M, et al. Myasthenia in pregnancy: best practice guidelines from a U.K. multispecialty working group. J Neurol Neurosurg Psychiatry 2014;85(5):538-543

11 Sommer N, Melms A, Weller M, Dichgans J. Ocular myasthenia gravis. A critical review of clinical and pathophysiological aspects. Doc Ophthalmol 1993;84(4):309-333

12 Bever CT Jr, Aquino AV, Penn AS, Lovelace RE, Rowland LP. Prognosis of ocular myasthenia. Ann Neurol 1983;14(5): 516-519

13 Zisimopoulou P, Brenner T, Trakas N, Tzartos SJ. Serological diagnostics in myasthenia gravis based on novel assays and recently identified antigens. Autoimmun Rev 2013;12(9): 924-930

14 Zisimopoulou P, Evangelakou P, Tzartos J, et al. A comprehensive analysis of the epidemiology and clinical characteristics of antiLRP4 in myasthenia gravis. J Autoimmun 2014;52:139-145

15 Tsivgoulis G, Dervenoulas G, Kokotis P, et al. Double seronegative myasthenia gravis with low density lipoprotein-4 (LRP4) antibodies presenting with isolated ocular symptoms. J Neurol Sci 2014;346(1-2):328-330 